\title{
Trigeminal neuralgia associated with osteogenesis imperfecta
}

A 21 year old man with severe osteogenesis imperfecta developed classic right maxillary trigeminal neuralgia. Brain imaging showed pronounced basilar invagination, which was probably responsible for the pain through distortion of the trigeminal sensory root secondary to displacement of the brainstem. Osteogenesis imperfecta may be added to Paget's disease as a potential cause of symptomatic trigeminal neuralgia in association with deformity of the skull base.

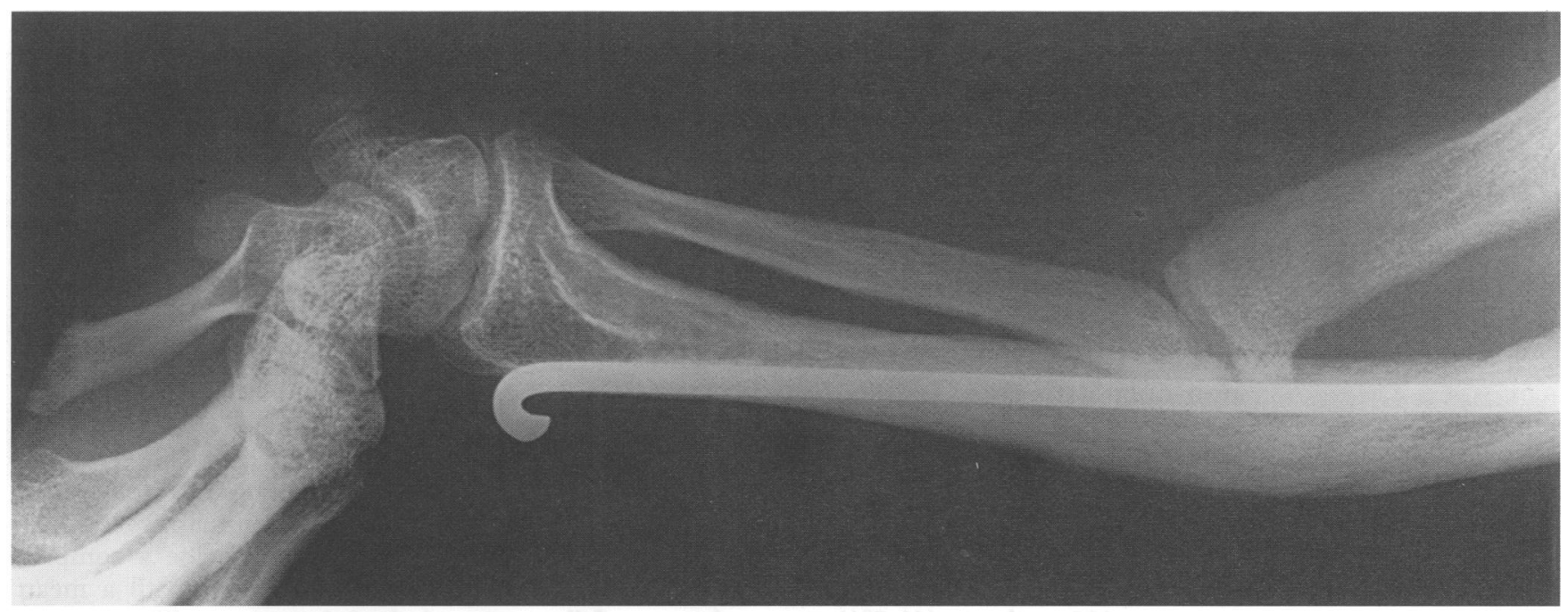

(A)
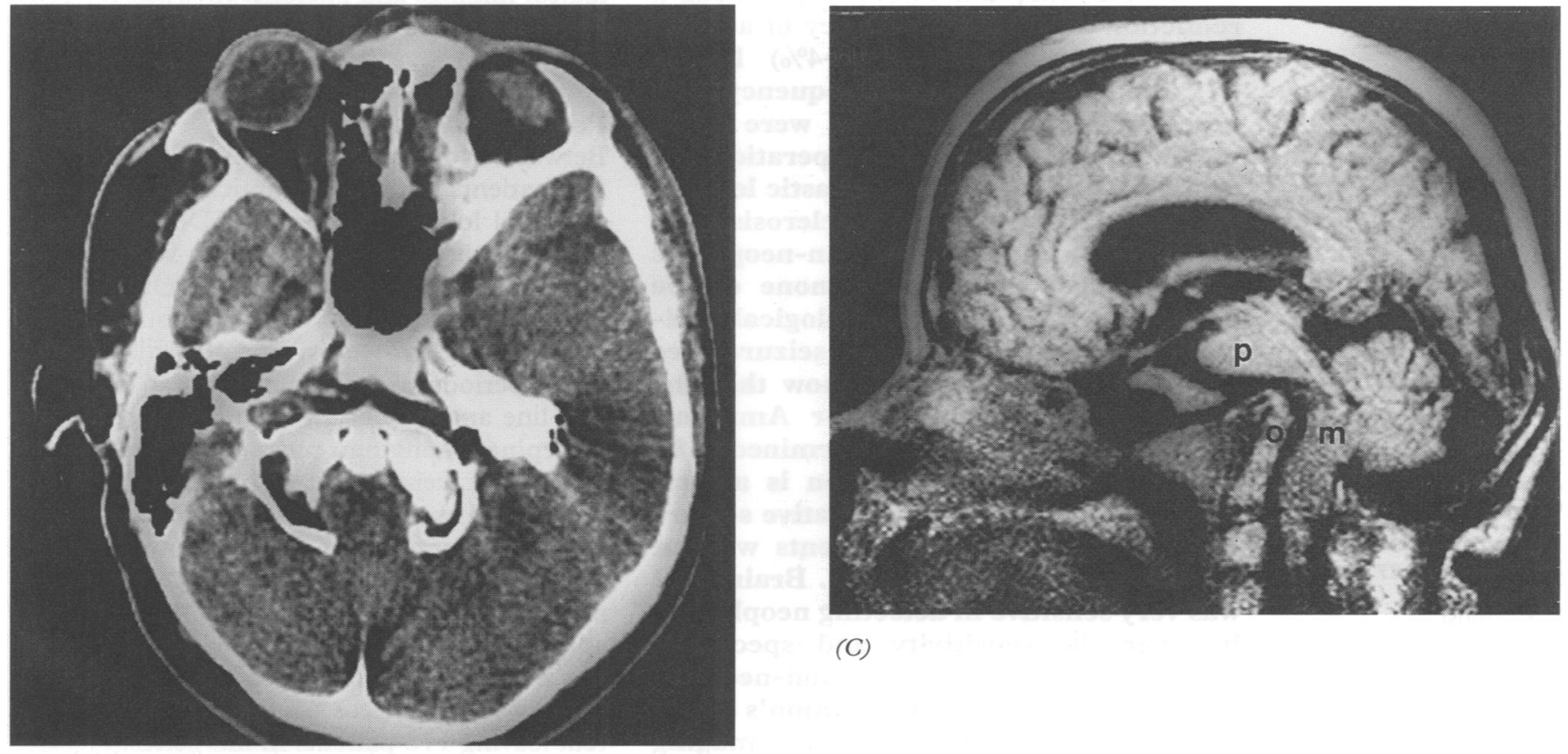

(C)

(B)

(A) Radiograph of left forearm showing evidence of osteogenesis imperfecta with an un-united fracture of the ulna and a healed fracture (and pin) in the radius. (B) CT of the head showing basilar invagination, with the odontoid process at the level of the upper petrous ridge. (C) Midline sagittal T1 weighted MRI of the cranium and craniocervical junction showing basilar invagination, the odontoid process encroaching on the anterior foramen magnum to produce pronounced compression of the anterior aspect of the pontomedullary junction. No other intracranial MR abnormality was detected; in particular no white matter lesions were seen. $P=$ Pons; $O=$ odontoid process; $M=$ medulla.

Correspondence to: Dr L Ginsberg, Department of Clinical Neurosciences, Royal Free Hospital School of Medicine, Rowland Hill Street, London NW3 2PF, UK. 